\title{
An Approach to Conformal Transformation Using Symbolic Language Facilities: Application in Electrical Engineering
}

\author{
Andrei Nicolaide \\ Department of Electrical Engineering and Applied Physics, Transilvania University of Brasov, \\ Boulevard Eroilor No. 29, 500036 Brasov, Romania \\ Correspondence should be addressed to Andrei Nicolaide, andrei.nicolaide@gmail.com
}

Received 21 December 2011; Accepted 23 January 2012

Academic Editors: A. Baïri, G. Fikioris, and F. Jauberteau

Copyright (C) 2012 Andrei Nicolaide. This is an open access article distributed under the Creative Commons Attribution License, which permits unrestricted use, distribution, and reproduction in any medium, provided the original work is properly cited.

\begin{abstract}
Several studies have been published on this subject concerning especially the calculation of the transformation integral and the plotting of the field spectrum. The method presented in this paper covers larger topics including field strength, circulation, and flux and offers solutions either in closed form or, if not possible, resorting to numerical integration and avoiding, for the considered cases, the utilization of any numerical method like the finite element method. In the present work, the usage of a symbolic language is presented and certain new results have been obtained. The method permits applying the conformal transformation avoiding the hand calculation of integrals for cases in which this calculation is very difficult by traditional procedures because it involves finding some changes of variable suitable for the considered case. For this purpose, the Maple 12 software has been used. An application for electrical engineering which has been considered led the author to find a new solution superior to the known ones.
\end{abstract}

\section{Introduction}

Conformal transformation is a very useful tool for the study of various potential fields. The procedure offers closed form solutions for many interesting cases, when it is possible, avoiding the utilization of any numerical method like the finite element method, finite difference method, and so forth. In the present work, the usage of a symbolic language, for this purpose, is presented. The procedure permits applying the conformal transformation avoiding the traditional hand calculation which involves some changes of variable difficult to be found and carried out. If the expression of the indefinite integral may not be obtained, the numerical integration is used. Therefore, the program permits controling the procedure, which may be difficult or even impossible by traditional calculation. For this purpose, we have considered and used the Maple 12 software. 
An application for electrical engineering for the calculation of the magnetic field in a rotating electrical machine has been considered. Simultaneously, some new results which show important differences relatively to the traditional treatments have been found.

\section{Recall of the Procedure of the Conformal Transformation}

Because in the literature the subject is treated in various manners, we will recall the principle and properties of conformal transformation in the manner we have used, for being able to pass consistently to the usage of symbolic programs in this field. Consider two complex planes, $z$ and $w$, respectively, and an analytic univalent function:

$$
w=f(z)
$$

Equation (2.1), for each value $z_{1}$ representing a point on plane $z$, yields a certain value $w_{1}$ representing a point on plane $w$. Analogously, to a succession of points on plane $z$, one obtains a succession of points on plane $w$, so that to any curve $z_{1} z_{2}$ on the $z$-plane, there corresponds a curve $w_{1} w_{2}$, which is called the transformed curve on the $w$-plane by (2.1).

Analogously, assuring the correspondence of the points on the two planes $z$ and $w$, an analytical function like (2.1) transforms any domain $\left(D_{z}\right)$ interior or exterior to a boundary on the $z$-plane, into a domain $\left(D_{w}\right)$ on the $w$-plane.

As known, a transformation like that above is called conformal transformation because two curves, which cross each other at a certain angle on plane $z$, keep the value and the sense of the angle, after their transformation on plane $w$.

As also known, at the base of the conformal transformation lies the Riemann theorem: the interior of a simply connected surface, of any domain $\left(D_{z}\right)$, can be represented in a biunivocal and conformal manner on the interior of a circle $\left(D_{w}\right)$, adopting three arbitrary constants.

In many applications it is necessary to make successively two conformal transformations, one from the $z$-plane into the upper half of the $w$-plane, the next from the upper halfplane into a rectangle domain $\left(D_{\zeta}\right)$ on a $\zeta$-plane.

\section{The Complex Potential Function and the Field Strength Components in Terms of the Abscissa of the $z$-Plane}

For any complex function as in the following expression:

$$
W(z)=U(x, y)+i V(x, y)
$$

assumed to be holomorphic in a certain domain, since the derivative with respect to the complex coordinate $z$ does not depend on the differentiation direction, there follows:

$$
\frac{d W}{\partial z}=\frac{d(U+i V)}{d(x)}=\frac{d(U+i V)}{d(i y)}
$$


and by equating the real and imaginary parts,

$$
\frac{\partial U}{\partial x}=\frac{\partial V}{\partial y}, \quad \frac{\partial U}{\partial y}=-\frac{\partial V}{\partial x} .
$$

The field strength, having on a plane $\zeta$ two components, may generally be expressed as follows:

$$
\underline{H}_{\zeta}=-\frac{\partial V}{\partial \xi}-i \frac{\partial V}{\partial \eta}=-\frac{\partial V}{\partial \xi}-i \frac{\partial U}{\partial \xi}=-i\left(\frac{\partial U}{\partial \xi}-i \frac{\partial V}{\partial \xi}\right)
$$

which with the above relations, and for simplicity, denoting $W=W_{\zeta}$, there follows

$$
\underline{H}_{\zeta}=-i\left(\frac{d W}{d \zeta}\right)^{*}
$$

For the $z$-plane, $W_{z}=W$ and it results that

$$
\underline{H}_{z}=-i\left(\frac{d W_{z}}{d z}\right)^{*}=\left(i \frac{\partial W_{\zeta}}{\partial z}\right)^{*}=\left(i \frac{d W_{\zeta}}{d \zeta} \frac{d \zeta}{d w} \frac{d w}{d z}\right)^{*}
$$

At each point of the domain $\left(D_{z}\right)$, there corresponds a certain value of the complex potential function expression $W=U+i V$. By conformal transformation of a domain $\left(D_{z}\right)$ into a domain $\left(D_{w}\right)$, at each point $w=w(z)$, obtained in domain $\left(D_{w}\right)$, there corresponds the same value of $W$ like in the corresponding point of domain $\left(D_{z}\right)$ above. Assume that the lines $V=$ const are equipotential lines and $U=$ const are lines of field. These lines, according to their expressions, are orthogonal lines and have the same meaning and expression in the corresponding two domains. One can write the expression of the potential difference between two points, denoted 1 and 2 in domain $\left(D_{z}\right)$ :

$$
\Delta V=V_{1}-V_{2}
$$

The potential difference between the corresponding points in domain $\left(D_{w}\right)$ is given by the same relation and should have the same value as above.

The flux through an arc of curve which ties two points denoted by 1 and 2 on domain $\left(D_{z}\right)$ will be

$$
\Delta \Psi=U_{2}-U_{1}
$$

The lines of flux crossing the arc of curve of above are delimited by the lines passing through points 1 and 2 .

For the sake of clearness, we can consider the tube of flux of a plane-parallel configuration crossing the mentioned arc of curve and having the thickness equal to unity. The described flux in domain $\left(D_{w}\right)$ is given by the same relation and should have the same value.

With the potential and flux being the same for the case of each of the two planes it is convenient to carry out the calculations in the case in which they are simpler. For instance, 
in many cases the most convenient case is that of a rectangular domain, say on a $\zeta$-plane, $\zeta=\xi+i \eta$, the upper and lower sides of the rectangle and the parallels to these are equipotential lines, whereas the vertical sides and the parallels to these are lines of field.

The passage from the upper half-plane to a rectangle is related to a strip, and is accomplished by the known conformal transformation

$$
\zeta=\ln (w) .
$$

Then, the calculation of circulation or flux is very easy.

In several applications, in Maple 12, it may be necessary to replace symbol $\zeta$ by $t$, the first being, in the mentioned software, protected. For practical applications, it is worth noting that if the potentials and fluxes keep their values after a conformal transformation, the quantities: electric capacitance, electric resistance, magnetic reluctance, also keep their values.

For practically solving a problem by using a conformal transformation, the following stages have to be browsed:

(1) To find the adequate analytic function for transforming the given domain configuration into another convenient for solving the problem, in most cases it is the case of the rectangle explained above. Sometimes, several transformations are necessary for this purpose.

(2) If inside the domain $\left(D_{w}\right)$ the complex potential is

$$
W=w(z)
$$

the same expression represents, apart from a constant, factor, or term, the complex potential in the interior of domain $\left(D_{z}\right)$.

(3) The computation of potential differences and fluxes should be performed using the complex potential function choosing that of the domains $\left(D_{w}\right)$ or $\left(D_{\zeta}\right)$, where the computations are easier. Using the complex potential function, the direct calculation of fluxes, used in traditional treatments, is no more required.

(4) In many cases it suffices to obtain the function for conformal transformation of the domain $\left(D_{z}\right)$ of the $z$-plane into the interior of a circle or of a rectangle, in a $\zeta$ plane, because it is easy to obtain their conformal transformation into the domain of the previously described rectangle, in the case of certain adequate boundary conditions. No general method exists for the conformal transformation formula, of any domain, but there are relations for particular cases very useful for many applications.

A very interesting case is that of the interior domain of a polygonal contour into the $z$-plane, with $n$ vertices, situated at finite distance. For any vertex with ordinal number $k$, the oriented angle denoted by $\beta_{k}$ with which the side has to be rotated for obtaining the direction of the next side should be given. Instead of the oriented angle, the value of the internal angle $\alpha_{k}$ at this vertex can be given, sometimes being easier for calculations. The following usual relations are fulfilled:

$$
-\pi<\beta_{k}<\pi \quad 0<\alpha_{k}<2 \pi .
$$


tion

The transformation of this domain into that of the upper half-plane is given by the rela-

$$
\frac{d z}{d w}=A \prod_{k=1}^{n}\left(w-u_{k}\right)^{-\beta_{k} / \pi} .
$$

There follows

$$
z=A \int \prod_{k=1}^{n}\left(w-u_{k}\right)^{-\beta_{k} / \pi} d w+C .
$$

A transformation of the form (3.12) or (3.13) is called, as known, a Schwarz-Christoffel transformation. Many treatments of this formula have been given in the literature, among which are [1-6]. However, certain cases, especially if each angle $\beta_{k}$ is not strictly positive [3], still may raise difficulties. Cases of this type can be found in [1].

\section{The Treatment of the Conformal Transformation Using the Maple Language}

For the previously mentioned cases, the known calculations are difficult and their following is also difficult. In these cases, the symbolic languages for programming allow the possibility of avoiding both difficulties. We will explain the method on an example known in literature, in order to permit an easy comparison. We have used the Maple 12 language.

The advantages are the following

(a) There is no need to look for the obtained expression, in closed form, of the integral, if it exists, the indefinite integral is automatically returned by the software, even if the expression is so long that the traditional solving could not be expected. Moreover, the display of the integral in closed form is not always necessary because the evaluation of the stored result for the given data is also automatically performed by the program. If the expression in closed form of the indefinite integral cannot be obtained, the program continues to work correctly as explained in Section 6.

(b) For the preparation of the program, it is convenient to keep unchanged the values of certain variables or names during the calculation, according to the required conditions. For this purpose, as we have experienced, the use of procedures (proc) is very useful.

(c) An important remark, as principle, is that for conformal transformations we must work not with physical lengths that should be expressed in metres but with the corresponding relative quantities. Therefore, the lengths which occur should be divided by another length, usually a segment which exists in the considered configuration.

As we have realized, the usage of a program prepared in Maple language permits avoiding the calculation of fluxes, which can be replaced by using the complex potential function that remains unchanged by conformal transformations. Therefore, it suffices to calculate the difference of two coordinates of two points for obtaining the flux passing through the respective tube of flux, in any transformed configuration. 
At the same time, for obtaining the point of the minimum value of the component of the field strength, it has been possible to calculate by Maple the derivative of the considered function, avoiding the classical treatments $[1,2]$.

As known, in classical treatments, the representation of the normal component of the field strength versus abscissa of the given configuration is relatively difficult. We succeeded in avoiding this complication by using a parametric plot by a procedure found in Maple 12 language.

It is useful to warn concerning calculations for the $w$-plane, because in the case of a parenthesis representing a square root, we can adopt one of the signs plus or minus, which cannot be automatically done, but only by tracking the continuity conditions. The program prepared by the author in Maple 12 language has permitted keeping the necessary conditions.

\section{The Considered Problem}

The problem chosen for presenting the procedure is the plane-parallel configuration of Figure 1. This configuration can also be considered as having a certain thickness, equal to unity. For fixing the ideas, the contour in the figure represents the traces of the boundary of the interior domain. The configuration is considered to be the model of certain physical objects. The continuous lines constitute the walls delimiting the margins (sides) of ferromagnetic pieces. The space delimited by these walls is air or vacuum. The dashed lines represent the way along which we can travel the domain boundary by circumventing the singular points.

The top continuous line is considered to be at any magnetic potential $V$, supposed to be positive. The down broken line, by a slot, is considered to be at magnetic potential zero.

The gap between the two armatures, upper and lower, called air-gap, has its minimum thickness denoted by $\delta$ and it is assumed constant, except the portion over the slot having the aperture breadth $b_{0}$ and assumed infinitely deep. Therefore, between the upper and lower armatures a potential magnetic field exists.

If the slot did not exist, the flux between the two armatures had any value $\Phi_{0}$, but, due to the existence of the slot, it would be smaller, having a certain value $\Phi_{0}-\sigma$. One of the problems is to find the difference of the two fluxes, namely, $\sigma$. This mathematical problem arose, the first time, for the calculation of the magnetic circuit of rotating electrical machines and was solved by Carter and minutely examined by Gibbs [1]. For this reason, a certain obtained coefficient is called Carter coefficient or factor. The solution has implied difficult calculations and demanded much preoccupation for determining the necessary transformations. Also it is worth noting that an analysis of the singular points has not been carried out.

By using, as already mentioned, a symbolic language, we obtained much easier the solution and did a deeper analysis. As a result, it may be mentioned that the values of the field strength do not differ from those of [1], but for the fluxes the difference is sensible. The problem is not only mathematically interesting, but a correct solution can be used for solving similar technical problems.

\section{The Used Formulae and the Maple Built Programs}

At the beginning, we consider that some mentions concerning the calculation of integrals would be very useful, as follows. Maple 12 can perform both literal and numerical 

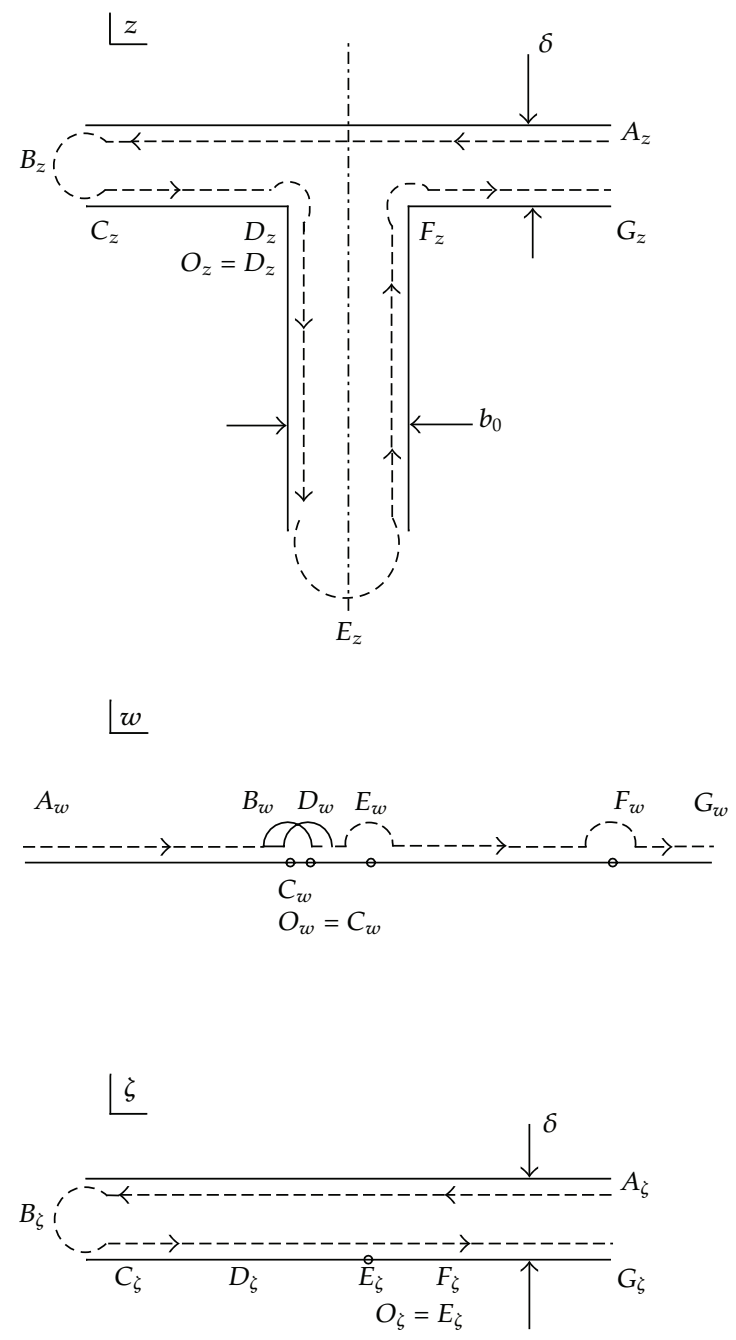

Figure 1: Configuration of a magnetic system.

integration, respectively. If Maple cannot find the expression, in closed form of the indefinite integral, or for definite integrals with one or both limits floating-point numbers (i.e., not fixed number of digits), for example, 3.04, 1e5, or an expression that leads to a floating-point number (value), the function call is returned, and then statement $\operatorname{eval} f(\operatorname{Int}(f, x=a . . b))$, where $f$ represents the integrand, $x$ the variable, and $a$ and $b$ the limits, computes the integral using numerical methods.

Before presenting the accurate solution we have obtained, it may be added that Maple allows also for a simple calculation of any flux, without implying the calculation in closed form of the occurring integral. It suffices to express the formula of the conformal transformation and the relation for determining the fluxes by using the expression of the complex potential function. For the limit zero, in the considered case, instead of the value zero, a negative value close to zero will be introduced for $c$. The Maple program returns the result very fast. However, we realized that this procedure is not always too precise. The reason is that the function which intervenes in the studied problem, when calculating the limit, shows 
relatively important oscillations in the vicinity of the singular point that reduce the precision of the result. However, the procedure may be useful in many problems as a fast and, in most cases, a satisfactory orientation.

The steps of the program will now be presented as follows.

The Schwarz-Christoffel transformation (3.12), for passing from the $z$-plane to the $w$ plane of Figure 1, is represented by one of the formulae given by the literal expression:

$$
\begin{gathered}
\frac{d z}{d w}=A \frac{\sqrt{w-a} \sqrt{w-b}}{w(w-1)}, \\
\frac{d z}{d w}=A \frac{\sqrt{w^{2}-(a+b) w+a \cdot b}}{w(w-1)} .
\end{gathered}
$$

In this case, the constants $a, b$ may not be determined by the general methods [3-6], because the angles $\beta_{k}$ are not all strictly positive. The constants have been determined by calculating certain distances of the figure by circumventing, along portions of circles, the each singular points (poles). However, we realized that these constants may be directly determined by replacing singular point circumventing integral with the calculation of the residue for the same point, for which the Maple soft gives a simple statement residue. We will give only the results using Algorithm 2:

$$
\frac{d z}{d w}=A \frac{\sqrt{w-a} \sqrt{w-b}}{w(w-1)}, \quad A=\frac{\delta}{\pi}, a=\frac{1}{b}
$$

and having in view that according to Figure $1, b>0$, we have

$$
\frac{d z}{d w}=A \frac{\sqrt{w^{2}-\left(b_{0}^{2}+2\right) w+1}}{w(w-1)}, \quad b_{0}=\frac{b-1}{\sqrt{b}} .
$$

In the case of Figure 2, the results are $b=4, a=0.25, b_{0}=1.5, \delta=1$.

In applying the above formula, when other requirements are not necessary, we will take the constant $A$ equal to unity, for simplicity.

In the program of Algorithm 1, the obtaining of the Schwarz-Christoffel solution is presented. As shown, the form is charged with many terms, but no effort is necessary for finding a simpler form, being not useful. It is worth noting that the solution of the transformation is given not directly, but using a procedure. The reason is to be able to change, at any step, the value of the variable, if necessary.

Remark 6.1. It is useful to note that in the program list, the comments are marked by the presence before them of the sign \# (also called pound sign) and the same sign is also placed before the considered input data. When pressing the ENTER key, the program returns the results without replacing the input data. If the input data are enabled, by removing the sign \#, the program returns the results corresponding to the respective input data. 


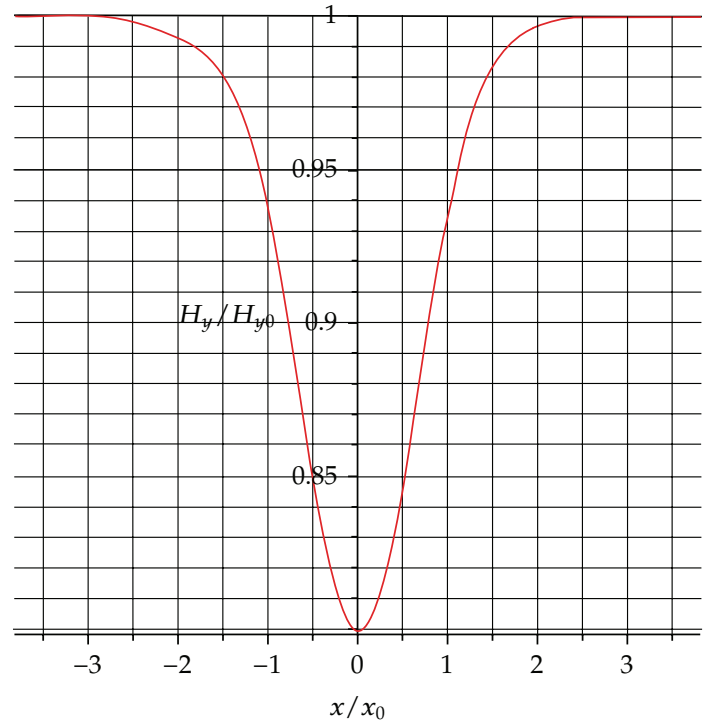

Figure 2: Normal component of the field strength for the case $b_{0}=1.5 \delta$.

\section{The Complex Potential and the Field Strength Components in the $z$-Plane}

We will begin by expressing the complex potential function for the case of a rectangle with particular boundary conditions described in Section 3. The complex potential can be expressed as follows.

The scalar potential, according to the described conditions, should be

$$
V(\xi, \eta)=\frac{V}{\delta} \eta, \quad i H_{\eta}=-i \frac{V}{\delta}
$$

and the flux on a portion $\xi$, considering the unit normal vector along the line of field, will be

$$
\Phi=\frac{V}{\delta} \xi
$$

The complex potential function will be

$$
\begin{gathered}
W_{\zeta}=U+i V=\frac{V}{\delta}(\xi+i \eta), \\
\underline{H}_{\zeta}=-i\left(\frac{d W_{\zeta}}{d \zeta}\right)^{*}=\left(i \frac{d W_{\zeta}}{d \zeta}\right)^{*} .
\end{gathered}
$$


\# An automatic generation of the sigma coefficient formula will be presented.

\# The fluxes between the point of abscissa $x w=-1$ and any point of abscissa $c$ will be considered.

\# In the program, both indexed names and symbols which are not indexed have been used.

\# The point $x w=-1$ is on the slot axis; on the line $A_{z} B_{z}$.

\# $b_{0}$ - the aperture breadth of the slot divided by the minimum air-gap thickness.

\# The reference field strength is that for the constant minimum air-gap thickness.

\# The involved relative field strength is its actual value divided by the reference value.

\# $\sigma$-flux difference due to the existence of the slot.

\# For obtaining the formula, the commands concerning the input variables should be disabled.

\# The multiplication sign is, in several versions, optional, a small space between factors suffices.

$P:=\operatorname{proc}(s)$

\# The considered transformation function will be:

$f:=(1 / \operatorname{eval} f(\pi)) \cdot\left(\sqrt{w^{2}-\left(b_{0}^{2}+2 .\right) \cdot w+1 . /}(w \cdot(w-1))\right) ;$

$z:=\operatorname{int}(f, w)$;

$z:=\operatorname{eval}(z, w=s)$;

end proc;

$F:=P(w)$;

\# $x w$-abscissa $w=-1$ in the $w$-plane.

$\# z_{1}$-complex vector potential function at point $w:=-1$. for the actual configuration.

$\# z_{2}$-complex vector potential function at point $w:=0$, hence a point $C_{w}$ for

the actual configuration.

$\# c$-abscissa of any point $c \in(-1,0)$, in particular 0 .

$\# b_{0}:=1.5$;

\# $x w:=-1$;

$\# c:=-0.1$

$z_{1}:=\operatorname{eval}(P(x w))$

$z_{1}:=\operatorname{eval} f\left(z_{1}\right)$;

$z_{2}:=P(c)$;

$z_{2}:=\operatorname{eval} f\left(z_{2}\right)$;

$z_{d}:=z_{2}-z_{1}$

$z_{d}:=\operatorname{simplify}\left(z_{d}\right)$;

$\# z_{d}$-gives the flux between the two points, calculated in the $w$-plane, in the case of actual field distribution in the $z$-plane.

$z_{t 1}:=\operatorname{eval} f((1 / \pi) \ln (x w))$;

$z_{t 2}:=\operatorname{eval} f((1 / \pi) \ln (c))$;

$z_{t}:=z_{t 1}-z_{t 2}$

$\# z_{t}$-gives the flux between the two points, calculated in the $w$-plane, in the case of a uniform field distribution in the $t$-plane (zeta-plane).

$\sigma:=z_{d}-z_{t}$;

$\sigma:=\operatorname{simplify}(\sigma)$

\# The total value will be $\sigma_{T}:=2 \sigma$.

\# Result after running the program:

$\# b_{0}:=1.5$;

\# $x w:=-1$;

$\# c:=-0.1$;

$$
\begin{aligned}
\sigma:=-\frac{1}{\sqrt{1}} & \left(0 . 3 1 8 3 0 9 8 8 6 1 \left(-1 . \ln (x w)+\ln (c)+\ln \left(-0.5000000000 b_{0}^{2}-1 .+c+\sqrt{c^{2}-1 . c b_{0}^{2}-2 . c+1 .}\right)\right.\right. \\
& -1 . \operatorname{arctanh}\left(\left(0.5000000000\left(-2 .+c b_{0}^{2}+2 . c\right)\right) / \sqrt{c^{2}-1 . c b_{0}^{2}-2 . c+1 .}\right) \\
& +b_{0}^{2} \ln \left(\left(-1 . b_{0}^{2}-1 . c b_{0}^{2}+2 . \sqrt{-1 . b_{0}^{2}} \sqrt{c^{2}-1 . c b_{0}^{2}-2 \cdot c+1 .}\right) /(c-1 .)\right) \\
& / \sqrt{-1 . b_{0}^{2}}-1 . \ln \left(-0.5000000000 b_{0}^{2}-1 .+x w+\sqrt{x w^{2}-1 . x w b_{0}^{2}-2 . x w+1 .}\right) \\
& +\operatorname{arctanh}\left(\left(0.5000000000\left(-2 .+x w b_{0}^{2}+2 . x w\right)\right) / \sqrt{x w^{2}-1 . x w b_{0}^{2}-2} \cdot x w+1\right) \\
& -1 . b_{0}^{2} \ln \left(\left(-1 . b_{0}^{2}-1 . x w b_{0}^{2}+2 \cdot \sqrt{-1 . b_{0}^{2}} \sqrt{x w^{2}-1 . x w b_{0}^{2}-2 \cdot x w+1 .}\right) /(x w-1 .)\right) / \\
& \left.\left.\sqrt{-1 . b_{0}^{2}}\right)\right)
\end{aligned}
$$


\# The results yielded by the formula obtained above and the enabled input data above:

$$
\begin{gathered}
b_{0}:=1.5 \\
x w:=-1 \\
c:=-0.1 \\
\sigma:=-0.1333736780-0 . I
\end{gathered}
$$

Algorithm 1: It is useful to recall that the pound sign (\#) marks that the characters following this sign represent a comment; therefore the software Maple does not process the above mentioned characters. For simplicity, in the algorithms of this article, the pound sign has been omitted when a sentence continues on several lines, but for running the program the sign must exist on every line.

\# The considered function for the calculation of residues will be:

$$
f:=A \cdot \sqrt{(w-a) \cdot(w-b)} /(w \cdot(w-1)) ;
$$

\# The symbols which do not occur in Algorithm 1, are explained below.

\# The curvilinear integral along the domain contour in direct sense corresponds to a semicircle inside the domain around the point considered singular point clockwise.

\# The residue is the curvilinear integral around the mentioned point divided by $2 \pi I$.

\# Therefore, the searched value will be obtained by multiplying the residue with $\pi I$ with the minus sign before.

\# The residues will be denoted by symbols of two letters, the first will be

$R$-abbreviation of residue, and the second letter will denote the point, like $G$, for which the residue will be calculated.

\# To calculate the distance between the points $G$ and $A$, we shall calculate the circulation along the semicircle at infinity. The corresponding residues at points $G, B, E$ will be:

$R G:=\operatorname{residue}(f, w=\infty+\infty I) ; R B:=\operatorname{residue}(f, w=0) ; R E:=\operatorname{residue}(f, w=1)$;

\# The corresponding lengths will be:

$L G:=A \pi I ; L B:=(\delta \sqrt{a \cdot b} / \pi) \pi I ; L E:=(\delta \sqrt{-b-a+1+a \cdot b} / \pi) \pi I ;$

$\#$ and there follows:

$L G:=\delta \sqrt{b+a+1-a \cdot b} ;$

\# But according to Figure 1:

$L G:=\delta I ; \quad L B:=\delta I ; \quad L E:=\delta \sqrt{b+a-1-a \cdot b} ; \quad L E:=b_{0} ;$

\# From the pair of relations $L B$, there follows that the the product $(a \cdot b)$ is equal to unity.

\# There follows:

$\#(b-1)^{2} / b=\left(b_{0} / \delta\right)^{2}$ and $b_{0} / \delta=(b-1) / \sqrt{b}$.

\# Using the details above, we obtain the relations of (6.2) and (6.3), where instead of $b_{0} / \delta$

we put simply $b_{0}$, namely the relative length, expressed as a ratio to base $\delta$.

Algorithm 2: Program for the calculation of the constants which occur in the conformal transformation.

Formula (3.6) will be used, and, for simplicity, it will be denoted $W=W_{\zeta}$, since it keeps the value after any conformal transformation, except a constant factor not generally necessary to be introduced. Then, for the $z$-plane, it results that

$$
\begin{gathered}
\underline{H}_{z}=-i\left(\frac{d W_{z}}{d z}\right)^{*}=\left(i \frac{\partial W_{\zeta}}{\partial z}\right)^{*}=\left(i \frac{d W_{\zeta}}{d \zeta} \frac{d \zeta}{d w} \frac{d w}{d z}\right)^{*}, \\
W_{\zeta}=\frac{V}{\delta}(\xi+i \eta) .
\end{gathered}
$$




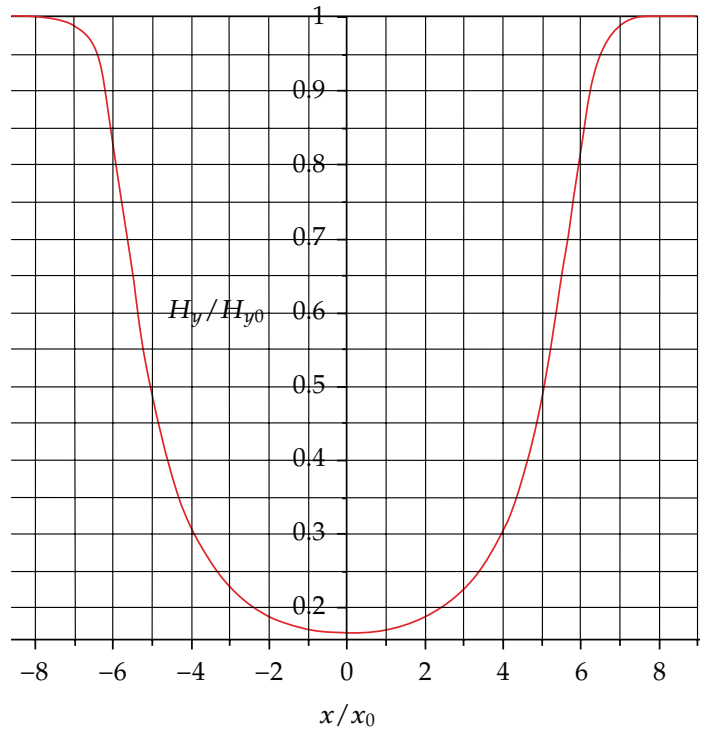

Figure 3: Normal component of the field strength for the case $b_{0}=12 \delta$.

There follows

$$
H_{x}=\operatorname{Re}\left(\underline{H}_{z}\right), \quad H_{y}=\operatorname{Im}\left(\underline{H}_{z}\right), \quad H_{y}=-\frac{V}{\delta} \cdot \frac{w-1}{\sqrt{w^{2}-\left(b_{0}^{2}+2\right) w+1}} .
$$

The minimum of $H_{y}$, according to the program of Algorithm 4, is reached for the value for which its derivative is zero. There follows that the axis of symmetry of the configuration passes through the point $w=-1$, which rends minimum the considered function.

\section{The Plotting of Curves}

Although the computation returns the values of the components in terms of $w$, and not of $x$, we avoided any difficulty by using the parametric plotting allowed by the Maple 12 software. We prepared the program of Algorithms 3 and 4.

The results may be seen in Figures 2 and 3, for two configurations. As expected, the minimum value of the normal component of the field strength is reached on the slot axis. Here is the case to mention that the called axis has the abscissa corresponding to $w=-1$.

\section{The Flux Difference Computing Program}

For the calculation of the flux reduction due to the existence of the slot that will be denoted by $\sigma$, we have to calculate both fluxes, the flux in the case in which the normal component of the field strength is considered having a constant value corresponding to the minimum value of the air-gap thickness, say $\Phi_{0}$, and that which actually exists for the real thickness, say $\Phi$. Both fluxes will be calculated using the complex potential function, separate flux calculation being no more necessary, at the difference of known works. 


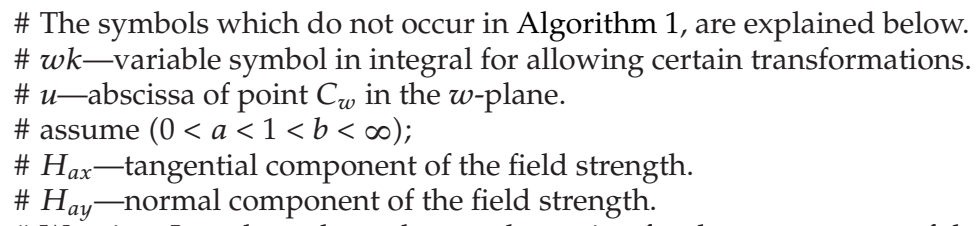

\# The symbols which do not occur in Algorithm 1, are explained below.

\# $w k$-variable symbol in integral for allowing certain transformations.

\# $u$-abscissa of point $C_{w}$ in the $w$-plane.

\# assume $(0<a<1<b<\infty)$;

\# $H_{a x}$-tangential component of the field strength.

\# $H_{a y}$-normal component of the field strength.

\# Warning. In order to keep the usual notation for the components of the field strength we have used the indexed names $H_{x}$ and $H_{y}$ on the figures, but in the program we are obliged to modify the indices by putting, for example, $H_{a x}$ and $H_{a y}$ as components along the axes, otherwise, if in the program the ordinate $y$ also exists, errors appear, and the obtained values have no more meaning. For avoiding this circumstance, it is also possible to use only symbols.

$a:=0.25$;

$b:=4$.;

$b_{0}:=(b-1) / \sqrt{b} ;$

$H_{y 0}:=1$;

$F:=\operatorname{proc}(s)$

general $z, s$;

\# The transformation function is:

$f:=(1 / \pi) \cdot \sqrt{w k^{2}-\left(b_{0}^{2}+2 .\right) w k+1} . /(w k \cdot(w k-1)$.

$z:=\operatorname{int}(f, w k)$;

$z:=\operatorname{eval}(z, w k=s)+0.5 I$;

\# The last imaginary constant has been introduced for to be in accordance with the configuration of Figure 1.

end proc;

$u:=F(-1)$;

$u:=\operatorname{eval}(u)$;

\# Co-ordinate introduced for to have the value zero of the $x$-abscissa at the point of the minimum of $H_{a y}$.

for $w$ from -0.01 by -0.25 to -1.25 do

$\operatorname{prin}(w)$;

$z:=F(w)$;

$x:=\mathfrak{R}(z-u)$;

$y:=\mathfrak{J}(z)$;

$H_{a x}:=\Re\left(\right.$ conjugate $\left.\left(\left((w-1.) / \sqrt{w^{2}-\left(b_{0}^{2}+2 .\right) w+1 .}\right) I\right)\right) ;$

$H_{a y}:=-\Im\left(\operatorname{conjugate}\left(\left((w-1.) / \sqrt{w^{2}-\left(b_{0}^{2}+2 .\right) w+1 .}\right) I\right)\right) ;$

\# The sign minus has been introduced because, according to the assumed conditions in $t$-plane (zeta plane), the field strength is up-down.

end do;

$\# \operatorname{plot}\left(\left[x, H_{a y}, w=-1 . .-100\right]\right)$;

\# plot $\left(\left[x, H_{a y}, w=-100 . .-1000\right]\right)$;

$\# \operatorname{plot}\left(\left[x, H_{a y}, w=-1000 . .-100000\right]\right)$;

\# $\operatorname{plot}\left(\left[x, H_{a y}, w=-1 . .-0.01\right]\right)$;

$\# \operatorname{plot}\left(\left[x, H_{a y}, w=-0.01 . .-0.001\right]\right)$;

\# plot $\left(\left[x, H_{\text {ay }}, w=-0.001 . .-0.00001\right]\right)$;

\# For plotting by using the same program, it suffices to disable the lines for $w$ from...till do by putting \# and enabling the plot statements by removing \#.

\# Results after running the program:

$$
\begin{aligned}
& a:=0.25 \\
& b:=4 . \\
& b_{0}:=1.500000000 \\
& H_{y 0}:=1
\end{aligned}
$$

Algorithm 3: Continued. 


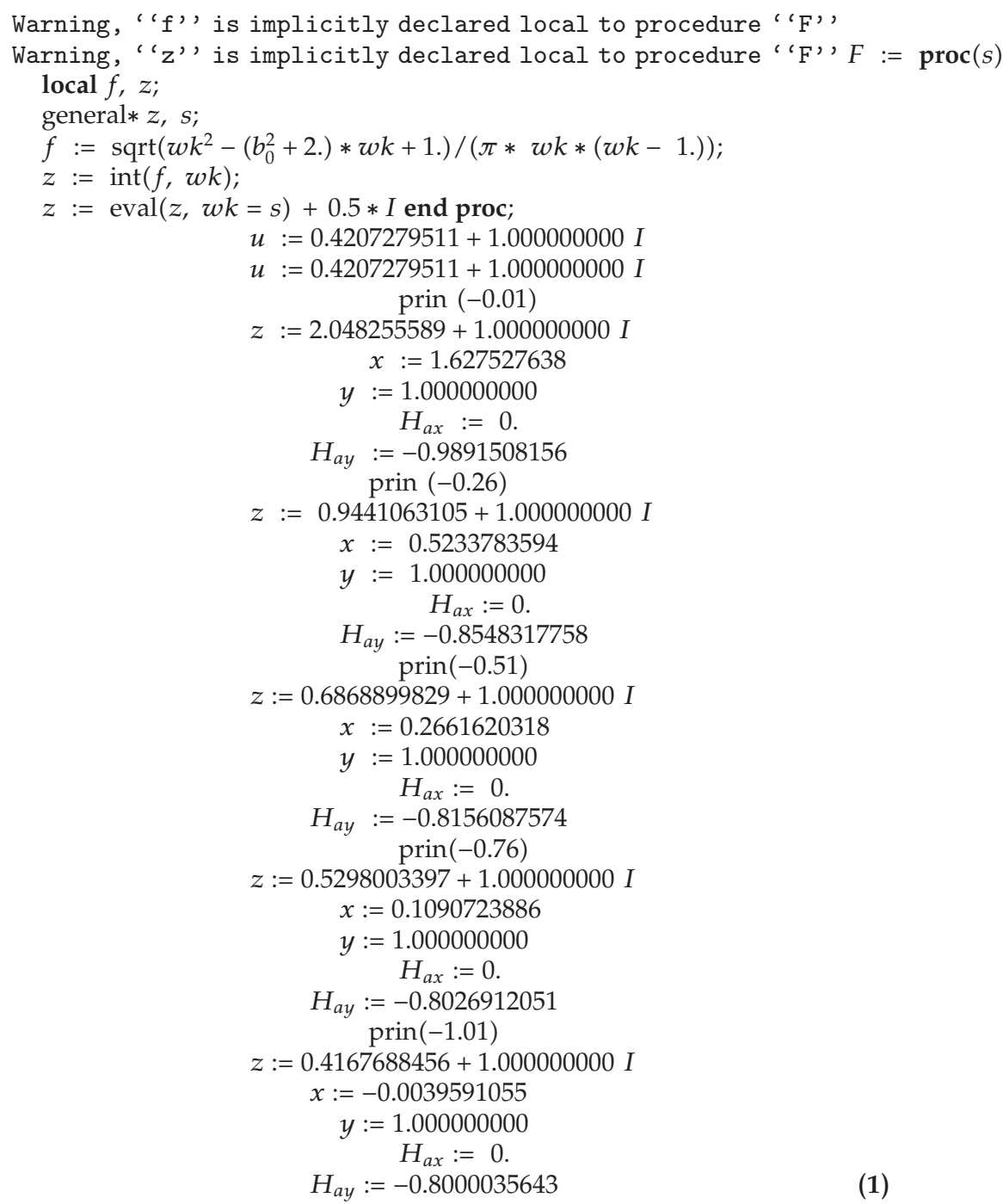

Algorithm 3: Program for calculating the normal component of the field strength versus the abscissa $x$, the origin of which is on the slot axis.

Due to the performed transformations, the calculation could refer to the $\zeta=\xi+i \eta$ plane domain, corresponding to the interval $w \in(-\infty,-1]$, that corresponds to the half of the length of the upper line (i.e., armature) with positive abscissa. The inverse values of $w$, for example, -0.001 and -1000 ., are symmetric values of $\xi$ with respect to $\xi=0$.

Remark 9.1. The last mention is of outstanding interest, not found in traditional treatments, because it means that to obtain the total flux it suffices to know only half of it, either for positive abscissa or for the negative abscissa. 


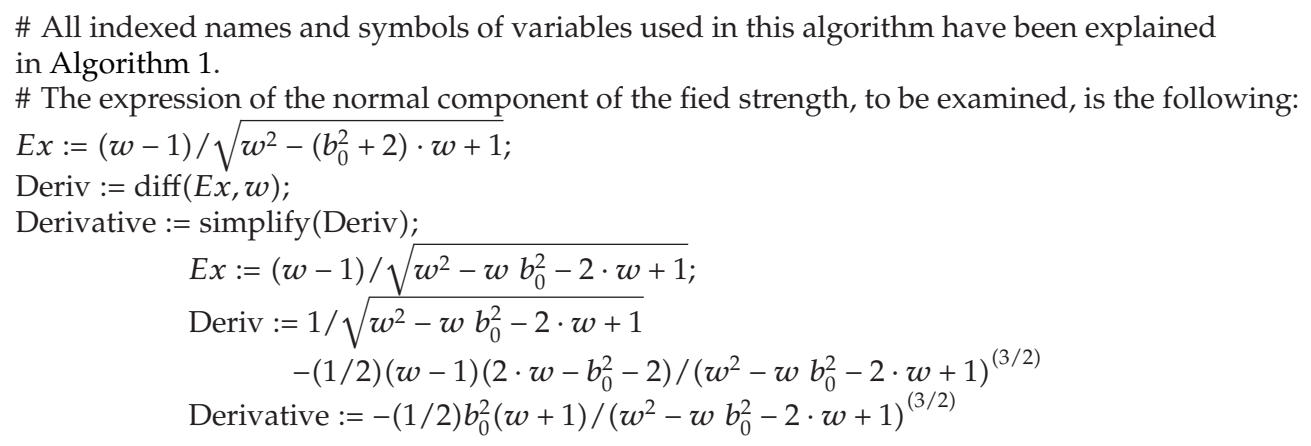

Algorithm 4: Program for the calculation of the derivative of the expression of the normal component of the field strength, with respect to the abscissa $w$, for obtaining the point where it is minimum.

In the case of infinite length as in [1], each of the two fluxes, $\Phi_{0}$ and $\Phi$, is infinite, but their difference is finite. For this reason, we have calculated the fluxes for the interval $w \in$ $[c,-1]$, the quantity $c$ being negative. Then, we have subtracted the results using the formula in closed form. Several terms containing the quantity $c$ reduced each other. For obtaining the fluxes, we have used the expression of the complex potential function. After having obtained the difference expression, we have considered the quantity $c$ approaching zero, being easier than for infinity. Here, the Maple software has presented a difficulty, because it could not remove the indeterminate form presented by the difference of the two terms, which occurred as the quantity $c$ is tending to zero. We have removed the indeterminate form of the difference of the two terms, by putting them in the form of a quotient and using the rule of de l'Hospital. Then, a relatively simple formula has been obtained. Both literal expression and numerical results have been obtained.

For easier solving of this and other cases, we have no more used the transformation formula based on relation (6.2), but (6.3). It has the advantage of returning the result expressed only in terms of the given data and leading to a simpler final literal expression.

The computing expressions we obtained may be followed in Algorithms 5 and 6, for both cases: $c \neq 0$ and $c=0$.

In this stage, the obtained formula can be used for one of the two purposes: to calculate the difference for a finite length of the armature but keeping the symmetry of the configuration, which cannot be performed by the results of the known literature, and for the case of the infinite length, given in the known literature, but surely, due to the mentioned circumstances, we obtained different results. Namely, for the relative value (i.e., length divided by $\delta$ and field strength divided by $H_{y 0}$ ), we obtained $\sigma_{T}=-0.330$, whereas by the traditional procedure the value is $\sigma_{T}=-0.220$.

\section{The Carter Factor}

In practice, one of the most important results is the Carter factor, which refers to an alternative succession of slots and teeth, the pitch, that is, the distance between the axes of two successive slots having any value $t$. The traditional derivation passes from the calculation of the infinite length of the pitch, as explained above to a finite length accepting very large approximations. 
\# The symbols which are supplementary to those of Algorithm 1 are expressed below and represent expressions in the calculation and have been put in a form to facilitate their usage. \# They are $y_{1}, y_{2}, y_{a}, y_{L}$.

\# The expression of $y_{a}$ is not necessary in usual calculations, but can serve to some verifications.

\# Another symbol to be added is $k$ and serves to establish the sign of the flux in terms of the used abscissa.

$b_{0}:=1.5$

$c:=-0.1$

$x w:=-1$;

$k:=\operatorname{signum}(\mathrm{c}+1)$;

$y_{1}:=(1 / 2.) \ln \left(1 . /\left(\sqrt{c^{2}-c b_{0}^{2}-2 . c+1}-0.5\left(-2 .+c b_{0}^{2}+2 . c\right)\right)\right)$;

$y_{2}:=-(1 / 2.) \ln \left(c^{2} /\left(\sqrt{c^{2}-c b_{0}^{2}-2 . c+1}+0.5\left(-2 .+c b_{0}^{2}+2 . c\right)\right)\right)$;

$y_{a}:=y_{1}+y_{2}$

$y_{a}:=\operatorname{arctanh}\left((1 . / 2)\left(-2 .+c b_{0}^{2}+2 . c\right) / \sqrt{c^{2}-c b_{0}^{2}-2 . c+1}\right)$;

$y_{L}:=-(1 / 2.) \ln \left(-8 . /\left(b_{0}^{2}+4.\right) b_{0}^{2}\right)$;

\# For $c=0$, in expression below, $y_{2}$ should be replaced by its limit $y_{L}$;

$\sigma:=-k\left(0.3183098861\left(\left(b_{0}^{2} / \sqrt{-b_{0}^{2}}\right) \ln \left(\left(-b_{0}^{2}-c b_{0}^{2}+2 \cdot \sqrt{-b_{0}^{2}} \sqrt{c^{2}-c b_{0}^{2}-2 \cdot c+1}\right) /(c-1).\right)\right.\right.$

$+\ln \left(-(1 . / 2) b_{0}^{2}+c-1 .+\sqrt{\left.c^{2}-c b_{0}^{2}-2 . c+1 .\right)}-\left(y_{1}+y_{2}\right)\right.$

$-\left(b_{0}^{2} / \sqrt{-b_{0}^{2}}\right) \ln \left(\left(-b_{0}^{2}-1 . x w b_{0}^{2}+2 \cdot \sqrt{-b_{0}^{2}} \sqrt{x w^{2}-x w b_{0}^{2}-2 \cdot x w+1}.\right) /(x w-1).\right)$

$-\ln \left((-1 . / 2) b_{0}^{2}+x w-1 .+\sqrt{x w^{2}-x w b_{0}^{2}-2 . x w+1}\right)$

$\left.\left.+\operatorname{arctanh}\left(0.5000000000\left(-2 .+x w b_{0}^{2}+2 . x w\right) / \sqrt{x w^{2}-x w b_{0}^{2}-2 . x w+1}\right)-\ln (x w)\right)\right)$;

$\sigma:=\operatorname{simplify}(\sigma)$ :

\# The total value will be $\sigma_{T}:=2 \sigma$.

\# For to keep the correct sign of the flux, even say for $c=-1000$, the signum function has been used.

\# Results after running the program:

$$
\begin{gathered}
b_{0}:=1.5 \\
c:=-0 \\
x w:=-1 \\
k:=1 \\
y_{1}:=-0.4398994358 \\
y_{2}:=0.1887092260-1.570796327 I \\
y_{a}:=-0.2511902098-1.570796327 I \\
y_{L}:=0.2820350692-1.570796327 I \\
\sigma:=-0.1333736806-1.10^{-10} I
\end{gathered}
$$

Algorithm 5: Program for calculating the fluxes in terms of the geometrical dimensions of the considered domain in the general case.

With the formulae we established previously, with much better approximation, we can calculate it. It is the ratio between the flux in the case in which the thickness of the air-gap was constant and equal to its minimum value and the actual flux in the real case. Both results from the complex potential function and the relations are given in the program of Algorithm 5. For example, we have considered the case $c=-0.001$ to which there corresponds $x=2.363674365$ relative units with respect to the air-gap thickness. 
\# Below, a special case will be examined and an indeterminate form will be removed. \# The symbols which are supplementary to those of Algorithm 1 are given below and represent expressions occurring in the calculation and have been put in a form for facilitating their usage.

\# They are $y_{1}, y_{2}, y_{a}, y_{L}$. Another symbol to be added is $k$ and serves to establish the sign of the flux in terms of the used abscissa.

$b_{0}:=1.5$

$c:=-0$;

$x w:=-1$;

$k:=\operatorname{signum}(\mathrm{c}+1)$;

$y_{1}:=(1 / 2.) \ln \left(1 . /\left(\sqrt{c^{2}-c b_{0}^{2}-2 . c+1}-0.5\left(-2 .+c b_{0}^{2}+2 . c\right)\right)\right)$;

$y_{2}:=-(1 / 2.) \ln \left(c^{2} /\left(\sqrt{c^{2}-c b_{0}^{2}-2 . c+1}+0.5\left(-2 .+c b_{0}^{2}+2 . c\right)\right)\right)$;

\# $y_{a}:=\operatorname{arctanh}\left((1 . / 2)\left(-2 .+c b_{0}^{2}+2 . c\right) / \sqrt{\left.c^{2}-c b_{0}^{2}-2 . c+1 .\right)}\right.$;

\# The command concerning the variable $y_{a}$ may be enabled only if $\mathrm{c}$ is different from zero. $y_{L}:=-(1 / 2.) \ln \left(-8 . /\left(b_{0}^{2}+4.\right) b_{0}^{2}\right)$;

\# For $c=0$, in expression below, $y_{2}$ should be replaced by its limit $y_{L}$;

$$
\begin{aligned}
& \sigma:=-k\left(0 . 3 1 8 3 0 9 8 8 6 1 \left(\left(b_{0}^{2} / \sqrt{-b_{0}^{2}}\right) \ln \left(\left(-b_{0}^{2}-c b_{0}^{2}+2 \cdot \sqrt{-b_{0}^{2}} \sqrt{c^{2}-c b_{0}^{2}-2 \cdot c+1} .\right) /(c-1 .)\right)\right.\right. \\
&+\ln \left(-(1 . / 2) b_{0}^{2}+c-1 .+\sqrt{\left.c^{2}-c b_{0}^{2}-2 . c+1 .\right)}-\left(y_{1}+y_{2}\right)\right. \\
&-\left(b_{0}^{2} / \sqrt{-b_{0}^{2}}\right) \ln \left(\left(-b_{0}^{2}-1 . x w b_{0}^{2}+2 \cdot \sqrt{-b_{0}^{2}} \sqrt{x w^{2}-x w b_{0}^{2}-2 . x w+1}\right) /(x w-1 .)\right) \\
&-\ln \left((-1 . / 2) b_{0}^{2}+x w-1 .+\sqrt{\left.x w^{2}-x w b_{0}^{2}-2 . x w+1 .\right)}\right. \\
&\left.\left.+\operatorname{arctanh}\left(0.5000000000\left(-2 .+x w b_{0}^{2}+2 . x w\right) / \sqrt{x w^{2}-x w b_{0}^{2}-2 . x w+1 .}\right)-\ln (x w)\right)\right) \\
& \sigma:=\operatorname{simplify}(\sigma): \\
& \text { \# The total value will be } \sigma_{T}:=2 \sigma . \\
& \text { \# } \text { Results after running the program: }
\end{aligned}
$$

$$
\begin{gathered}
b_{0}:=1.5 \\
c:=-0 . \\
x w:=-1 \\
k:=1 \\
y_{2}:=\text { Float (undefined) + Float (undefined) } I \\
y_{L}:=0.2820350692-1.570796327 I \\
\sigma:=-0.1651915504-1.10^{-10} I
\end{gathered}
$$

Algorithm 6: Program for calculating the fluxes in terms of the geometrical dimensions of the considered domain in a special case avoiding the occurring indeterminate form.

According to the above definition, the Carter factor will be given by the formula:

$$
k_{C}=\frac{z_{d}}{z_{t}}, \quad z_{d}=z_{t}+\sigma,
$$

where, according to Algorithms 1 and $2 z_{d}$ (in program the symbols are $z_{d}$ and $z_{t}$ ) represents the length on the over armature, on the $z$-plane, between the points corresponding to abscissae $w=-1$ and $w=c$, for all $c<0$ on the $w$-plane, and $z_{t}$ represents the length on the upper armature on the zeta-plane which is just the flux between the two points corresponding to those above on the $w$-plane. Both quantities are proportional, apart from a constant factor, to the complex potential function. For the case of Figure 2, using formula (10.1) and the results of Algorithms 1 and 5, we have obtained $k_{C}=1.07496873$. As a verification, in the ideal case for $c \rightarrow 0$, for all $c<0$, there follows $k_{C} \rightarrow 1$. 


\section{Comparison with Other Programs}

In the literature there are several Maple programs concerning the Schwarz-Christoffel transformation, mainly devoted to the calculation of the transformation and to plotting the field spectrum.

An interesting analysis on the Schwarz-Christoffel transformation has been presented in [7], also resulting in the preparation of a Maple program for the calculation of the integral. In many applications of the field analysis it does not suffice being necessary the calculation of potential differences (circulations) and fluxes. Just this subject has made one of the purposes of the present work, resulting in Maple programs we have applied in practice.

Moreover, we have established that, in several cases we have experimented, numerical integration leads to a precision very near to that by using the solution in closed form. As explained, the fluxes and circulations have been calculated avoiding the classical procedures which involve supplementary integrations.

\section{Conclusion}

A new method, efficient in many cases, for using the conformal transformation for the calculation of potential fields has been presented. The method is based on the utilization of symbolic languages and permits to directly obtain the integral of the Schwarz-Christoffel formula, regardless of its complication, provided the solution in closed form exists, which is not possible by the traditional methods. The method correctly works even if the expression in closed form of the indefinite integral cannot be obtained. Moreover, the proposed procedure has allowed the obtaining of new results of higher accuracy than previously. Also, the analysis of the singular point could be carried out and taken into consideration.

\section{References}

[1] W. J. Gibbs, Conformal Transformations in Electrical Engineering, Chapman \& Hall, London, UK, 1958.

[2] A. Nicolaide, Masini Electrice. Teorie. Proiectare (Electrical Machines. Theory. Design), vol. 1, Scrisul Romanesc, Craiova, Romania, 1975.

[3] A. Nicolaide, "Numerical methods in conformal transformation," Proceedings of the Institution of Electrical Engineers, vol. 124, no. 2, pp. 1110-1111, 1977.

[4] A. Nicolaide, "Conformal transformation using direct minimization methods," Revue Roumaine des Sciences Techniques. Série Électrotechnique et Énergétique, vol. 23, no. 3, pp. 373-380, 1978.

[5] A. Nicolaide, Bazele fizice ale electrotehnicii (Physical Foundations of Electrical Engineering), vol. 1, Scrisul Romanesc, Craiova, Romania, 1983.

[6] T. A. Driscoll and L. N. Trefethen, Schwarz-Christoffel Mapping, vol. 8 of Cambridge Monographs on Applied and Computational Mathematics, Cambridge University Press, Cambridge, UK, 2002.

[7] P. P. Bergonio, Schwarz-Christoffel transformations, M.S. thesis, Graduate Faculty of the University of Georgia Southwestern State University, Athens, Ga, USA, 2003. 


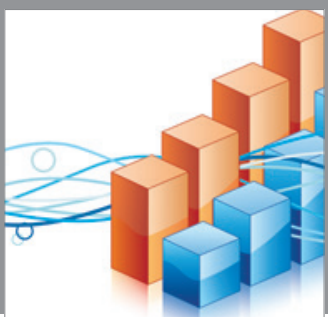

Advances in

Operations Research

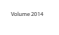

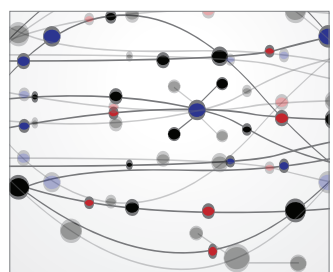

\section{The Scientific} World Journal
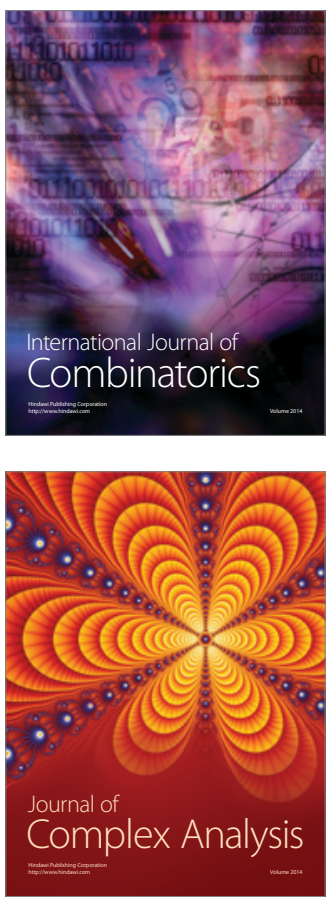

International Journal of

Mathematics and

Mathematical

Sciences
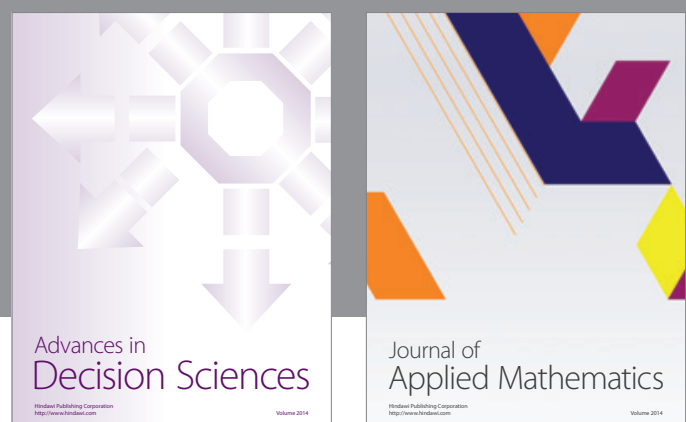

Journal of

Applied Mathematics
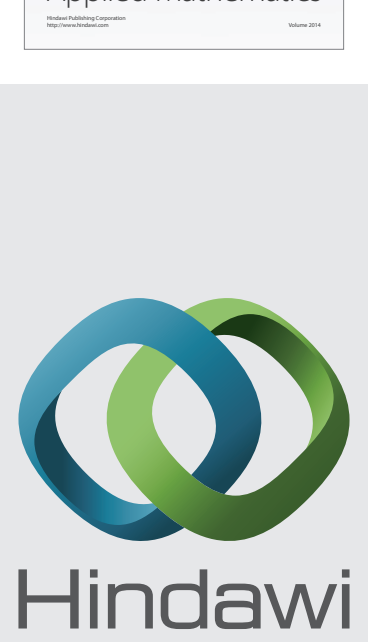

Submit your manuscripts at http://www.hindawi.com
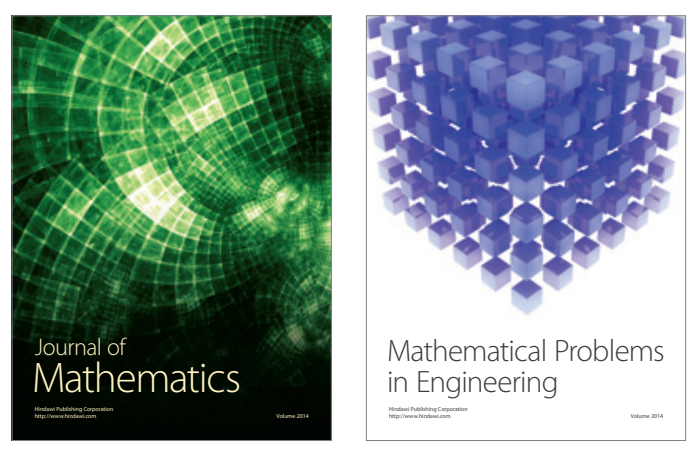

Mathematical Problems in Engineering
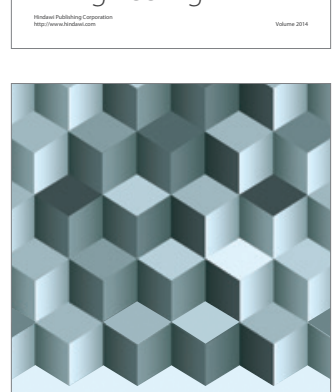

Journal of

Function Spaces
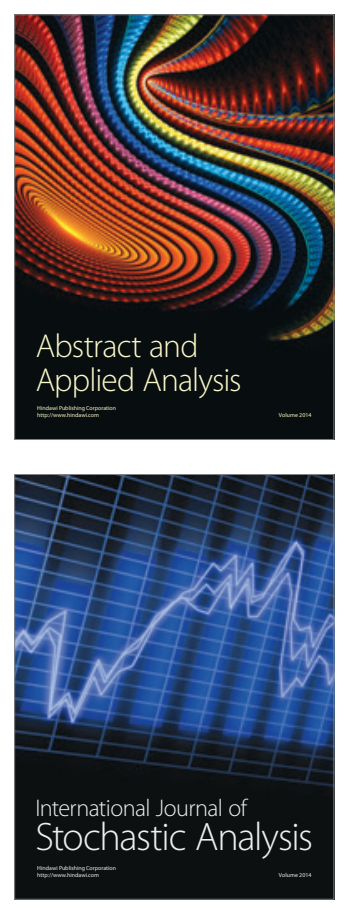

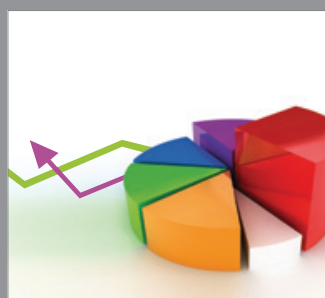

ournal of

Probability and Statistics

Promensencen
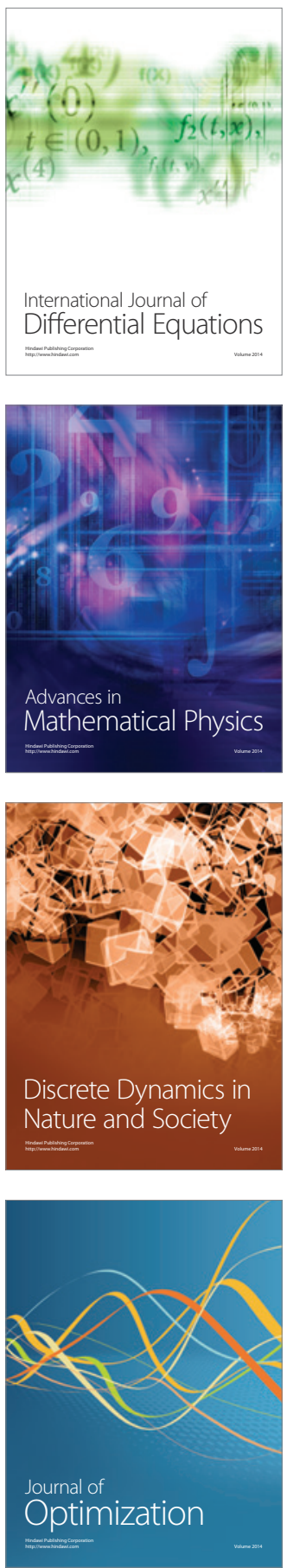